\title{
Gestational Trophoblastic Neoplasia: A Tunisian Multicenter Study
}

\author{
Haifa Rachdi ${ }^{1}$, Amina Mokrani ${ }^{1}$, Rim Batti ${ }^{1}$, Henda Raies ${ }^{1}$, Omar Touhami ${ }^{2}$, \\ Mouna Ayadi ${ }^{1}$, Khadija Meddeb ${ }^{1}$, Feryel Letaief ${ }^{1}$, Yosra Yahiaoui ${ }^{1}$, Nesrine \\ Chraiet $^{1}$, Amel Mezlini ${ }^{1}$
}

${ }^{1}$ Department of Medical Oncology at Salah Azaiz institute, Tunis, Tunisia. ${ }^{2}$ "CC" Department of Obstetrics and Gynecology, Tunis Maternity and Neonatology Center, Tunis, Tunisia.

\begin{abstract}
Gestational trophoblastic disease (GTD) develops from abnormal cellular proliferation of trophoblasts following fertilization. It includes benign trophoblastic disease (hydatidiform moles (HM) and the malignant trophoblastic diseases or gestational trophoblastic neoplasia (GTN). The frequency of the GTD in Tunisia is one per 918 deliveries. The aim of this study is to analyze the clinical characteristics, treatment and outcomes of GTD at Salah Azaiez Institute (ISA). Medical records of women diagnosed with GTD at ISA from January 1, 1981 to December 31, 2012 were retrospectively reviewed. FIGO (International Federation of Gynecology and Obstetrics) score was determined retrospectively for patients treated before 2002 One hundred and nine patients with GTN were included. Patients presented with metastases at $43 \%$ of cases. The most common metastatic sites was lung (30\%) and vagina (13\%). 56 (51\%) patients had low-risk and 21 (19\%) cases had high-risk, the FIGO score was not assessed in 32 cases. After a median follow-up of 46 months, 21 patients were lost to follow-up, 12 patients died, 19 progressed and 8 relapsed. At 10 years, the OS rate was $85 \%$ and the PFS rate $79 \%$. OS was significantly influenced by the presence of metastases at presentation (M0 $100 \%$ vs. Metastatic $62 \% ; \mathrm{p}<0.0001$ ), FIGO stage (I-II 100\% VS 61\% and 65\% for stage III and IV; $p<0.001$ ), FIGO score (low-risk $99 \%$ vs. high-risk $78 \%$; $p$ $<0.001)$. GTN is a significant source of maternal morbidity with increased risk of mortality from complications if not detected early and treated promptly.
\end{abstract}

Keywords: Gestational trophoblastic disease- Choriocarcinoma- Outcomes- prognosis

Asian Pac J Cancer Care, 4 (2), 59-64

\section{Introduction}

Gestational trophoblastic disease (GTD) develops from abnormal cellular proliferation of trophoblasts following fertilization. It includes benign trophoblastic disease (hydatidiform moles (HM) and the malignant trophoblastic diseases or gestational trophoblastic neoplasia (GTN). The frequency of the GTD in Tunisia is one per 918 deliveries [1].

The aim of this study was to analyze the clinical characteristics, treatment and outcomes of GTD at Salah Azaiez Institute (ISA).
Submission Date: 12/16/2018 Acceptance Date: 02/09/2019

\section{Materials and Methods}

Medical records of women diagnosed with GTD at ISA from January 1, 1981 to December 31, 2012 were retrospectively reviewed. Patients with incomplete records were excluded from the study. Disease diagnosis, treatment, follow-up data, overall survival (OS) and progression free survival (PFS) were analyzed. FIGO score was determined retrospectively for patients treated before 2002. The data processing and analysis were carried out using the SPSS software version 20. Survival probabilities were estimated using Kaplan-Meier method. 126 patients were reported and included during 31 years (1981-2012). Of these cases, 17 (13\%) were diagnosed hydatidiform mole and were excluded from this study. 109

Corresponding Author:

Dr. Haifa Rachdi

Department of medical oncology at Salah Azaiz institute, Tunis, Tunisia.

Email: haifa.rachdi@yahoo.fr 
patients presented with GTN and were included.

\section{Results}

During the study period, 109 patients presented with GTN and were included. Their ages ranged between 18 and 53 years with an average of 34 years. Consanguinity was found in 33 cases ( $45 \%$ first degree). The median delay to diagnosis was 3 months (0-36). Sixty eight patients presented with amenorrhea followed by bleeding per vaginally or abortion followed by irregular bleeding per vaginally. GTN occurred after full-term pregnancy in two cases (after one and 14 months). Initial presenting features and reproductive history are summarized in (Table 1).

Performance status (PS) was $=0$ in $35 \%$ (38 pts), $\mathrm{PS}=1$ in $31 \%$ (34 pts), $\mathrm{PS}=2$ in $11 \%$ (12 pts) and $\mathrm{PS}=3$ in 1 case. Ultra sound imaging was performed in all cases, only 72 reports were available. The typical snow storm appearance was described in 29 cases, heterogeneous mass in 30 cases (mean tumor size $=68 \mathrm{~mm}$ ), increase in uterine size in 10 cases and there was an intraperitoneal effusion in three cases.

Histological evidence was obtained in 66 cases. It was obtained by suction dilation and curettage in 41 cases, hysterectomy specimen in 16 cases and a biopsy of a metastasis in nine cases. Histology confirmed 43 cases of choriocarcinoma and 23 of invasive mole. In the other cases the diagnosis was retained on clinical, biological and radiological data.

At diagnosis, 53 patients (48\%) had localized disease (M0), whereas 43 had metastases and 13 were classified $\mathrm{Mx}$.

The most common metastatic sites at initial diagnosis were the lungs (33 cases), and vagina (15 cases). Three patients had brain metastasis, one patient hepatic metastasis, one patient had a sub diaphragmatic mass of $12 \mathrm{~cm}$, one patient had suspicious pelvic nodes and one patient had a biceps muscle metastasis.

The mean $\beta \mathrm{HCG}$ (Human chorionic gonadotropin) level before treatment was $152170 \mathrm{UI} / 1$ (median 10 068).

According to FIGO (International Federation of Gynecology and Obstetrics) stage, 56 patients were stage I, 4 patients were stage II, 30 patients were stage III and 8 patients stage IV; The FIGO Stage was not evaluable in 11 cases.

According to FIGO 2002 scoring system, 56 cases were scored low risk and 21 high risk, the FIGO score was not evaluable for 32 patients.

\section{Chemotherapy}

In the low-risk group, 24 patients received MTX and 6 patients received ACT-D. Among high-risk patients, 13 received Cisplatin based regimen associated with actinomycin or adriamycin and etoposide, 1 patient did not receive cisplatin because of renal failure, 1 patient received BEP regimen and one patient received EMACO regimen.

Patients treated before the WHO scoring system received different chemotherapy regimen,
Table 1. Initial Presenting Features and Reproductive History

\begin{tabular}{|c|c|c|}
\hline & $\mathrm{N}(\%)$ & \\
\hline \multicolumn{3}{|l|}{ Age at first intercourse } \\
\hline Median & 22 & \\
\hline Min & 15 & \\
\hline Max & 32 & \\
\hline \multicolumn{3}{|l|}{ Age at menarche } \\
\hline Mean & 13 & \\
\hline Min & 10 & \\
\hline Max & 17 & \\
\hline \multicolumn{3}{|l|}{ Past medical history } \\
\hline Prior Hydatidiform mole & 11 & \\
\hline Prior spontaneous miscarriage & 50 & \\
\hline $\begin{array}{l}\text { Voluntary interruption of } \\
\text { pregnancy }\end{array}$ & 7 & \\
\hline \multicolumn{3}{|l|}{ Gravidity } \\
\hline 0 & 4 & \\
\hline 1 & 6 & \\
\hline 2 & 9 & \\
\hline$\geq 3$ & 60 & \\
\hline Median & 4 & \\
\hline \multicolumn{3}{|l|}{ Parity } \\
\hline 0 & 15 & \\
\hline 1 & 12 & \\
\hline 2 & 9 & \\
\hline$\geq 3$ & 51 & \\
\hline Median & 3 & \\
\hline \multicolumn{3}{|l|}{ Number of children } \\
\hline 0 & 11 & \\
\hline 1 & 12 & \\
\hline 2 & 11 & \\
\hline$\geq 3$ & 45 & \\
\hline \multicolumn{3}{|l|}{ Contraception } \\
\hline Estrogen/progestin & 14 & \\
\hline intrauterine device & 5 & \\
\hline No contraception & 9 & \\
\hline Unkown & 81 & \\
\hline \multicolumn{3}{|l|}{ Initial presenting features } \\
\hline Abnormal vagina bleeding & 68 & \\
\hline Abdominal pain & 6 & \\
\hline Metastases & 8 & \\
\hline $\begin{array}{l}\text { During the monitoring after } \\
\text { molar pregnancy }\end{array}$ & 10 & \\
\hline Disease category/CT & Number of patient & $\begin{array}{l}\text { CRI } \\
\mathrm{N}\end{array}$ \\
\hline \multicolumn{3}{|l|}{ Low risk } \\
\hline Single agent Methotrexate & 24 & 17 \\
\hline Single agent Actinomycin & 6 & 4 \\
\hline \multicolumn{3}{|l|}{ Combination chemotherapy } \\
\hline MTX-vincristine & 6 & 6 \\
\hline Actinomycin-etoposide & 16 & 8 \\
\hline Cisplatin-adriamycin-etoposide & 1 & 1 \\
\hline Cisplatin-actino-etoposide & 1 & 1 \\
\hline Unkown & 2 & \\
\hline
\end{tabular}


Continued Table 1. Initial Presenting Features and Reproductive History

\begin{tabular}{|c|c|c|}
\hline & Number of patient & $\begin{array}{c}\text { CRI } \\
\mathrm{N}\end{array}$ \\
\hline \multicolumn{3}{|c|}{ High Risk Combination chemotherapy } \\
\hline $\begin{array}{l}\text { Cisplatin-actinomycin- } \\
\text { etoposide }\end{array}$ & 8 & 6 \\
\hline $\begin{array}{l}\text { Cisplatin-adriamycin- } \\
\text { etoposide }\end{array}$ & 3 & 2 \\
\hline Cisplatin adriamycin & 1 & unkown \\
\hline Etoposide-actinomycin & 2 & 0 \\
\hline Cisplatin actinomycin & 1 & 0 \\
\hline BEP & 1 & 1 \\
\hline EMACO & 1 & \\
\hline \multicolumn{3}{|l|}{ Single agent $\mathrm{CT}$} \\
\hline Actionomycin & 2 & 0 \\
\hline Methotrexate & 1 & 0 \\
\hline Unkown & 1 & \\
\hline \multicolumn{3}{|c|}{ Patients with not assessed FIGO score } \\
\hline \multicolumn{3}{|l|}{ Single agent $\mathrm{CT}$} \\
\hline Methotrexate & 8 & \\
\hline \multicolumn{3}{|l|}{ Combination CT } \\
\hline Actinomycin-etoposide & 5 & \\
\hline Methotrexate-oncovin & 8 & \\
\hline Actinomycin-vinblastin & 2 & \\
\hline Methtrexate-vinblastine & 1 & \\
\hline EP/EMA & 1 & \\
\hline BEP & 1 & \\
\hline Cisplatin-actino-etoposide & 1 & \\
\hline $\begin{array}{l}\text { Cisplatin-actinomycin } \\
\text {-bleo-velbe-Endoxan }\end{array}$ & 1 & \\
\hline Unkown & 4 & \\
\hline
\end{tabular}

the decision of the type of chemotherapy was based on only clinical features. When retrospectively assessed, we noted that in four cases of high risk FIGO score patients received single agent chemotherapy and 24 patients with low-risk received combination agent chemotherapy. Treatment modalities are detailed in Table 2.

Treatment toxicity totally happened in 50 patients. Grade 2 stomatitis was observed in 19 patients treated with MTX. There were two events of transient generalized skin rush after MTX single agent treatement.

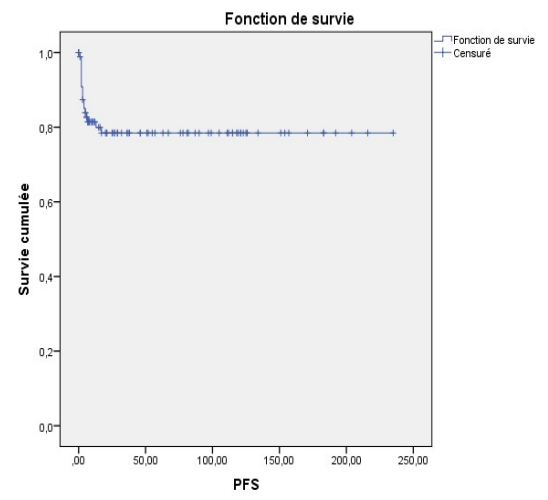

Figure 1. Kaplan-Meier Estimates of Overall Survival

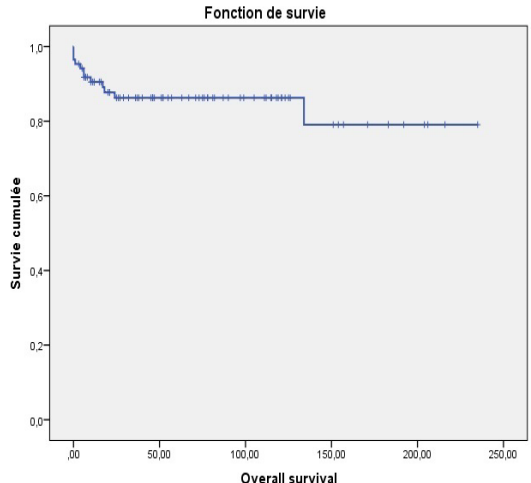

Figure 2. Kaplan-Meier Estimates of Progression Free Survival

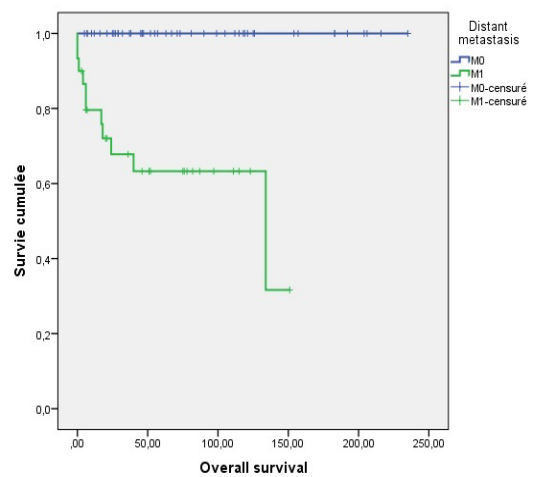

Figure 3. Kaplan-Meier Estimates of Overall Survival by Distant Metastasis

Five $(4 \%)$ patients had grade IV toxicity and four patients died due to chemotherapy toxicity, all of them happened after cisplatine based chemotherapy. One patient had renal failure, two patients had grade IV febrile neutropenia, one patient had hepatic toxicity and one patient died after anaphylactic reaction after Cisplatine perfusion.

\section{Surgery}

Eighteen patients underwent initial surgery, 4 patients had uterine perforation and 14 had excessive uterine bleeding. One patient had initial surgery for a biceps muscle metastasis. Five patients were operated after chemotherapy for residual disease.

\section{Radiotherapy}

Two patients had radiotherapy, one for brain metastasis and the other a hemostatic radiotherapy for a bleeding vaginal metastasis.

\section{Outcome and follow up}

The response to treatment was evaluated during follow-up by clinical examination, $\beta$ HCG levels and imaging as and when required. 21 patients were lost to follow-up and were excluded from survival analysis.

After a median follow-up from initial diagnosis of 46 months, 72 patients achieved complete response, 12 patients died (eight due to disease progression and four 
Table 2. Summary of Chemotherapeutic Protocols. Legend, CRI, Complete Response to Initial Chemotherapy

\begin{tabular}{|c|c|c|}
\hline Disease category/CT & $\begin{array}{c}\text { Number of } \\
\text { patient }\end{array}$ & $\begin{array}{l}\text { CRI } \\
\mathrm{N}\end{array}$ \\
\hline \multicolumn{3}{|l|}{ Low risk } \\
\hline Single agent Methotrexate & 24 & 17 \\
\hline Single agent Actinomycin & 6 & 4 \\
\hline \multicolumn{3}{|l|}{ Combination chemotherapy } \\
\hline MTX-vincristine & 6 & 6 \\
\hline Actinomycin-etoposide & 16 & 8 \\
\hline Cisplatin-adriamycin-etoposide & 1 & 1 \\
\hline Cisplatin-actino-etoposide & 1 & 1 \\
\hline Unkown & 2 & \\
\hline \multicolumn{3}{|l|}{ High Risk Combination chemotherapy } \\
\hline Cisplatin-actinomycin-etoposide & 8 & 6 \\
\hline Cisplatin-adriamycin-etoposide & 3 & 2 \\
\hline Cisplatin adriamycin & 1 & unkown \\
\hline Etoposide-actinomycin & 2 & 0 \\
\hline Cisplatin actinomycin & 1 & 0 \\
\hline BEP & 1 & 1 \\
\hline EMACO & 1 & \\
\hline \multicolumn{3}{|l|}{ Single agent $\mathrm{CT}$} \\
\hline Actionomycin & 2 & 0 \\
\hline Methotrexate & 1 & 0 \\
\hline Unkown & 1 & \\
\hline \multicolumn{3}{|l|}{ Patients with not assessed FIGO score } \\
\hline \multicolumn{3}{|l|}{ Single agent $\mathrm{CT}$} \\
\hline Methotrexate & 8 & \\
\hline \multicolumn{3}{|l|}{ Combination CT } \\
\hline Actinomycin-etoposide & 5 & \\
\hline Methotrexate-oncovin & 8 & \\
\hline Actinomycin-vinblastin & 2 & \\
\hline Methtrexate-vinblastine & 1 & \\
\hline EP/EMA & 1 & \\
\hline BEP & 1 & \\
\hline Cisplatin-actino-etoposide & 1 & \\
\hline Cisplatin-actinomycin-bleo-velbe- Endoxan & 1 & \\
\hline Unkown & 4 & \\
\hline
\end{tabular}

due to chemotherapy toxicity), 19 patients progressed to initial chemotherapy and eight patients relapsed.

At 10 years, OS was $82 \%$. The mean OS was 196 months IC95\% [175-218] (Figure 1).

Mean PFS was 185 months IC95\% [164-205], at 10 years PFS rate was $79 \%$ (Figure 2).

OS was significantly influenced by the presence of metastases at presentation (M0 $100 \%$ vs. Metastatic $62 \% ; \mathrm{p}<0.0001$ ) (Figure 3). Patients with advanced stage disease had poor survival when compared to the early stage group (I 100\%, II $95 \%$ vs. III $62 \%$, IV $62 \%$; $\mathrm{p}=0.001)$ (Figure 4). Patients with high-risk prognostic score compared to the low risk group showed poorer outcome (low-risk $99 \%$ vs. high-risk $76 \%$; $p=0.003$ ) (Figure 5).

After achieving treatment, 13 conceptions were reported resulting in no molar pregnancy: 3 miscarriage

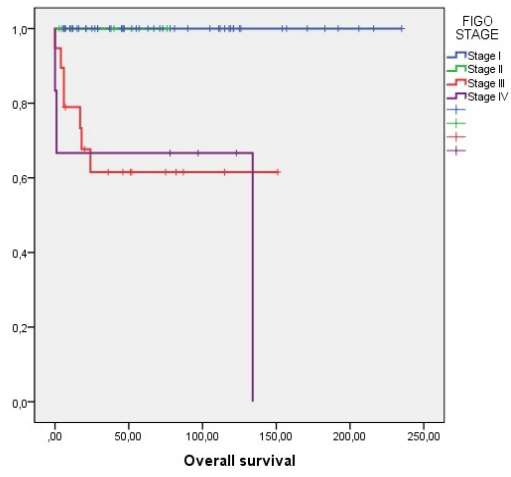

Figure 4. Kaplan-Meier Estimates of Overall Survival by FIGO Stage

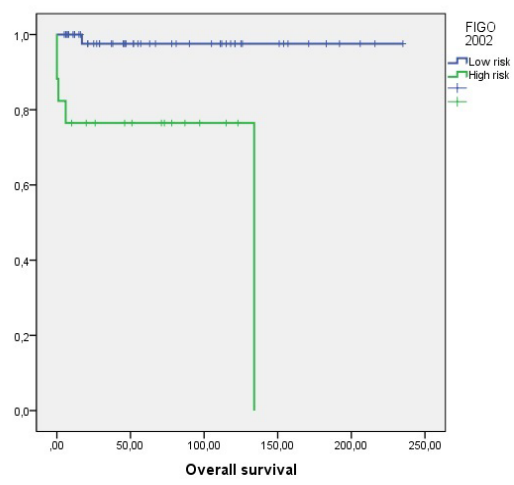

Figure 5. Kaplan-Meier Estimates of Overall Survival by FIGO Prognostic Score

and 10 full-term pregnancies. The mean time to pregnancy after achieving treatment was 37 months (range 8-123 months). Nine patients had full-term healthy births and a still birth in one case. One patient had repeated artificial insemination failure.

\section{Discussion}

GTD is a heterogeneous rare group of clinical conditions characterized by disordered differentiation and/or the proliferation of trophoblastic epithelium. GTN can arise after any type of pregnancy and can develop months or years after prior pregnancy. It affects women in the age group of 20 to 30 years. In our study, the median age was 34 years which is in comparison to peer reviewed published literature [2]. An increased risk of GTN have been linked to hormonal factors such as light menstrual flow, a menarche after 12 years-old, the use of oral contraceptive [3]. Other potential risk factors have also been reported for example; blood group A, past history of hydatidiform mole and maternal age [4]. GTN is a highly vascular disease and taking a biopsy of lesions are extremely risky. Histological confirmation is not essential before commencing chemotherapy. However, it may be useful to confirm diagnosis and obtain genetic analysis. Genotyping will be helpful to determine the causative pregnancy in patients with multiple pregnancies 
and to distinguish GTN from non gestational tumor [5]. The BHCG measurement is essential for diagnosis and management of GTD. Some assays can lead to false negative or false positive results because HCG can exist in different forms in patients with GTN. It's essential that the HCG assay can measure all forms of HCG [3]. Since 2002, the management of GTN is guided by the FIGO prognostic scoring. Patients with high risk disease had $0 \%$ chance to be cured with a single-agent chemotherapy. One recent study re-evaluated all prognostic risk factors involved in the FIGO scoring system in 813 patients with GTN and proposed a simplified alternative using only five factors [6].

GTN most frequently spreads to the lung. In our study, vaginal metastases were present in 13\%. In reported series vaginal metastases are present in $4-30 \%$ in GTN [7-8].

Patients with low-risk GTN can usually be treated successfully with single-agent chemotherapy. In our series of 56 low-risk patients, we observed a complete remission rate of $70 \%$ to single-agent Methotrexate and $66 \%$ to single-agent Dactinomycin. A randomized clinical trial compared biweekly Dactinomycin versus weekly Methotrexate demonstrated a superior response rate for Dactinomycin over Methotrexate (69 vs. 53\%; p $=0.015$ ) [9]. Retrospective reviews of patients with low-risk GTN treated with single-agent chemotherapy showed significantly higher primary remission rate with Dactinomycin than with Methotrexate regimen $[10,11]$. Due to decreased cost and the good tolerance of Methotrexate, especially for not inducing hair loss, Methotrexate was preferred as first line treatment. However a recent study suggest that women with hCG $>400000 \mathrm{UI} / 1$ are unlikely to be cured by single-agent chemotherapy and should be treated by multi-agent chemotherapy as first line treatment [12].

Among the patients with high-risk GTN, our data support the effectiveness of the combination chemotherapy. The preferred regimen in our study was the actinomycin, cisplatin and etoposide French regimen. The complete response rate with this regimen was $94.7 \%$ and the five-year OS $97 \%$. This regimen is highly active as first-line and as treatment for persistent/recurrent GTN [13]. Several regimens were developed. EMACO is used worldwide and is the optimal primary treatment because of short term toxicity and effectiveness [14]. A Korean study demonstrated a higher remission rate $(90.6 \%)$ with EMACO when compared with others regimens [15]. Patients with advanced disease may benefit from initial low dose chemotherapy to reduce early deaths [16].

Our study confirms the lower outcomes in patients with advanced-stage disease, metastatic disease and high-risk prognostic score [11-17].

The overall worldwide survival rate of low-risk GTN group is $100 \%$, and $80-90 \%$ for high-risk GTN group [11]. The overall survival rate for patients with GTN treated at our institute was $85 \%$.

The reproductive success rates and gestational complications have become a concern for women treated for GTD. EMACO regimen may induce menopause three years earlier but fertility is not affected and $83 \%$ of women became pregnant after Methotrexate or EMACO [3]. The number of studies in the literature regarding this theme is small. The studies highlight that there is no change in fertility [18]. Under close monitoring, patients may conceive six months after achieving complete response [19]. One study demonstrated a slight increase in stillbirth after GTN treatment [20]. In our study, conception occurred resulting in no molar pregnancy, miscarriage and full-term pregnancy.

In conclusion, GTN is a significant source of maternal morbidity with increased risk of mortality from complications if not detected early and treated promptly. Management of Gestational disease should ideally be done in a specialized multi-disciplinary environment.

\section{Abbreviations}

GTD, Gestational trophoblastic disease.

GTN, Gestational trophoblastic neoplasia.

HM, Hydatidiform moles.

ISA, Salah Azaiez institute.

OS, Overall survival.

PFS, Progression free survival.

FIGO, International Federation of Gynecology and

Obstetrics.

PS, Performans status.

BHCG, Human chorionic gonadotropin.

MTX, methotrexate.

BEP, Bleomycine - Etoposide - platine.

EMACO, Etoposide - MTX- actinomycine-

cyclophosphamide- vincristine.

\section{What is already know on this topic}

- Gestational trophoblastic neoplasia are malignant disorders of invasive mole, choriocarcinoma, and the rare placental-site trophoblastic tumour. Overall cure rates can exceed $98 \%$. This success can be explained by the development of effective treatments and centralisation of care.

\section{What this study adds}

- Report a Tunisian experience with gestational trophoblastic neoplasia

- The results of this study can help to encourage a specialized multi-disciplinary environment for the management of Gestational disease.

\section{Declarations}

\section{Acknowledgements}

Not applicable.

\section{Funding}

Analysis funded by the Salah Azaiez Cancer Institute.

Availability of data and materials

The datasets used and/or analysed during the current study are available from the corresponding author on reasonable request. 


\section{Authors'contributions}

Haifa Rachdi, Amina Mokrani and Rim Batti performed acquisition of patient data, analysis and interpretation of patient data.

Henda Raies developed the study conception and design.

Omar Touhami ,Mouna Ayadi, Khadija Meddeb,Feryel Letaief, Yosra Yahiaoui, Nesrine Chraiet, Amel Mezlini performed critical revision of the manuscript.

All authors have read and agreed to the final version of this manuscript.

\section{Consent for publication}

Not Applicable.

\section{Ethics approval and consent to participate}

Approval was obtained from Salah Azaiez Institute to participate to the study.

\section{Competing interests}

The authors declare no competing interest.

\section{References}

1. Ben Ayed F et al. Placental tumors in Tunisia. Apropos of 35 cases. Tunis Med. 1986;64(2):137-41.

2.Sita-Lumsden A et al. Treatment outcomes for 618 women with gestational trophoblastic tumours following a molar pregnancy at the Charing Cross Hospital, 2000-2009. Br J Cancer. 2012;107(11):1810-4.

3. Seckl MJ, Sebire NJ, Berkowitz RS. Gestational trophoblastic disease. The Lancet. 2010;376(9742):717-29.

4. Strohl AE, Lurain JR. Clinical Epidemiology of Gestational Trophoblastic Disease. Curr Obstet Gynecol Rep. 2014;3(1):40-3.

5. M. J. Seckl et al. Gestational trophoblastic disease: ESMO Clinical Practice Guidelines for diagnosis, treatment and follow-up. Ann Oncol 2013; 24 (Suppl 6):39-50

6. Eysbouts YK et al. Can the FIGO 2000 scoring system for gestational trophoblastic neoplasia be simplified? A new retrospective analysis from a nationwide dataset. Ann Oncol. 2017;28(8):1856-1861

7. Berry E, Hagopian GS, Lurain JR. Vaginal metastases in gestational trophoblastic neoplasia. J Reprod Med. 2008;53(7):487-92.

8. Cagayan MSFS. Vaginal metastases complicating gestational trophoblastic neoplasia. J Reprod Med. 2010;55(5-6):229-35.

9. Osborne RJ et al. Phase III trial of weekly methotrexate or pulsed dactinomycin for low-risk gestational trophoblastic neoplasia: a gynecologic oncology group study. J Clin Oncol. 2011;29(7):825-31.

10. Matsui H, Iitsuka Y, Seki K, Sekiya S. Comparison of chemotherapies with methotrexate, VP-16 and actinomycin-D in low-risk gestational trophoblastic disease. Remission rates and drug toxicities. Gynecol Obstet Invest. 1998;46(1):5-8.

11. Al-Husaini $\mathrm{H}$ et al. Gestational trophoblastic neoplasia: treatment outcomes from a single institutional experience. Clin Transl Oncol. 2015;17(5):409-15.

12. McGrath $\mathrm{S}$ et al. The management and outcome of women with post-hydatidiform mole 'low-risk' gestational trophoblastic neoplasia, but hCG levels in excess of 100000 IU/1. Br J Cancer. 2010;102(5):810-4.

13. Even $\mathrm{C}$ et al. Actinomycin D, cisplatin, and etoposide regimen is associated with almost universal cure in patients with high-risk gestational trophoblastic neoplasia. Eur J Cancer. 2014;50(12):2082-9.

14. Deng L, Zhang J, Wu T, Lawrie TA. Combination chemotherapy for primary treatment of high-risk gestational trophoblastic tumour. Cochrane Database Syst Rev. 2013;(1):CD005196.

15. Kim SJ et al. Management of high-risk hydatidiform mole and persistent gestational trophoblastic neoplasia: the Korean experience. J Reprod Med. 2007;52(9):819-30.

16. Alifrangis $\mathrm{C}$ et al. EMA/CO for high-risk gestational trophoblastic neoplasia: good outcomes with induction lowdose etoposide-cisplatin and genetic analysis. J Clin Oncol. 2013;31(2):280-6.

17. Yanaranop M, Potikul C, Tuipae S. A 10-Year Clinical Experience of Gestational Trophoblastic Disease at Rajavithi Hospital, 2001-2010. J Med Assoc Thail. 2016;99 Suppl 2:S17-27.

18. Garcia MT, Lin LH, Fushida K, Francisco RPV, Zugaib M. Pregnancy outcomes after chemotherapy for trophoblastic neoplasia. Rev Assoc Medica Bras. 2016;62(9):837-42.

19. Brown J, Naumann RW, Seckl MJ, Schink J. 15 years of progress in gestational trophoblastic disease: Scoring, standardization, and salvage. Gynecol Oncol. 2017;144(1):200-7.

20. Berkowitz RS, Tuncer ZS, Bernstein MR, Goldstein DP. Management of gestational trophoblastic diseases: subsequent pregnancy experience. Semin Oncol. 2000;27(6):678-85.

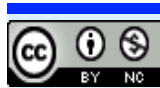

This work is licensed under a Creative Commons AttributionNon Commercial 4.0 International License. 